\title{
Correction: Identification of a novel Wilson disease gene mutation frequent in Upper Austria: a genetic and clinical study
}

\section{Harald Hofer • Claudia Willheim-Polli • Peter Knoflach · Christian Gabriel • Wolfgang Vogel • Michael Trauner •} Thomas Müller · Peter Ferenci

Published online: 16 June 2021

(C) The Author(s), under exclusive licence to The Japan Society of Human Genetics 2021

Correction to: Journal of Human Genetics

https://doi.org/10.1038/jhg.2012.65

The original version of this article unfortunately contained a mistake. In the article, we described a "new" ATP7B variant (p.R816S) as a common mutation in Upper Austria, mistakenly considering the last 3 nucleotides of exon 9 to code for Arginine. In fact, the third nucleotide is the first of the adjacent intron. Reanalysis of the sample by NGS showed that this variant was in fact $2447+1 \mathrm{G}>\mathrm{T}$. This variant is listed as likely pathogenic in ClinVar (https://www.ncbi.nlm.nih.gov/clinvar/). 